\title{
Research Article Symmetries of $n$ th-Order Approximate Stochastic Ordinary Differential Equations
}

\author{
E. Fredericks ${ }^{1}$ and F. M. Mahomed ${ }^{2}$ \\ ${ }^{1}$ Department of Mathematics and Applied Mathematics, University of Cape Town, Room 310.1, \\ Rondebosch 7700, South Africa \\ ${ }^{2}$ Centre for Differential Equations, Continuum Mechanics and Applications, School of Computational and \\ Applied Mathematics, University of the Witwatersrand, Johannesburg 2050, South Africa
}

Correspondence should be addressed to F. M. Mahomed, fazal.mahomed@wits.ac.za

Received 26 September 2012; Accepted 9 November 2012

Academic Editor: Asghar Qadir

Copyright (c) 2012 E. Fredericks and F. M. Mahomed. This is an open access article distributed under the Creative Commons Attribution License, which permits unrestricted use, distribution, and reproduction in any medium, provided the original work is properly cited.

Symmetries of $n$ th-order approximate stochastic ordinary differential equations (SODEs) are studied. The determining equations of these SODEs are derived in an Itô calculus context. These determining equations are not stochastic in nature. SODEs are normally used to model nature (e.g., earthquakes) or for testing the safety and reliability of models in construction engineering when looking at the impact of random perturbations.

\section{Introduction}

The modelling power of a SODE has been applied to many diverse fields of research, from the modelling of turbulent diffusion to neuronal activity in the brain. Models such as these are often influenced by more than one Wiener process. In these models, we assume that the Wiener processes are independent of one another. As a result of this increase in the number of Wiener processes affecting the model, the form of the Itô formula is slightly different to the one used in Fredericks and Mahomed [1,2]. The Itô formula is able to relate an arbitrary sufficiently smooth function $F(t, x)$ of time and space to a particular SODE, of which it is a solution. This formula, however, needs the SODE of the spatial random process $X(t, \omega)$ which drives the arbitrary function $F(X(t, \omega), \omega)$. The application of an SODE to an approximate analysis algorithm has been done by Ibragimov et al. [3] for scalar SODEs of first order. We extend this work for higher dimensions and order. We derive a similar conditioning on the temporal infinitesimal $\tau$ as had been performed by Ünal [4] and Fredericks and Mahomed [2]. We introduce operators to write the determining equations in a neater form. 
Wafo Soh and Mahomed [5] gave an algorithm to obtain Lie point symmetries for both first- and $n$ th-order SODEs. We briefly review their work and follow up with an extension from point symmetries to generalized symmetries.

The first section begins with the transformations of the spatial, temporal, and Wiener variables for an $n$ th-order Itô process. These transformations have the same properties as stated in Fredericks and Mahomed [6].

Using the Itô formula in conjunction with the infinitesimal transformations which preserve form invariance, we derive conditions for $n$ th-ordered SODEs that ensure the recovery of invariance preserving finite transformations from the infinitesimal ones. This has not been done in the past.

This is followed up with the development of recursive relations needed for finding the prolonged spatial infinitesimals in a SODE context by using the concept of form invariance. This differs from the methodology used by [5], where the recursive relation defined was predefined from an ODE context. As a result we also derive a conditioning on these prolonged spatial infinitesimal variables. We further derive a conditioning on the diffusion coefficient of the temporal generalised symmetry $\tau$, which is similar to that of Ünal [4]. We conclude the article with an introduction of operators which generalize the determining equations for any order SODE that is adaptable to both point and generalized symmetries.

The symmetries of high-ordered multidimensional stochastic ordinary differential equations (SODEs) are found using form invariance arguments on both the instantaneous drift and diffusion properties of the SODEs. We then apply this work to a generalized approximation analysis algorithm. The determining equations of SODEs are derived in an Itö calculus context. We also use the random time change formula used by Wafo Soh and Mahomed [5] to transform the Wiener processes.

\section{Review of Wafo Soh and Mahomed [5] for $n$ th-Order SODEs}

An $n$ th-order Itô process has the following vector form:

$$
\begin{gathered}
d \mathbf{X}^{(n-1)}(t)=\mathbf{f}\left(t, \mathbf{X}(t), \dot{\mathbf{X}}(t), \ldots, \mathbf{X}^{(n-1)}(t)\right) d t+\mathbf{G}\left(t, \mathbf{X}(t), \dot{\mathbf{X}}(t), \ldots, \mathbf{X}^{(n-1)}(t)\right) d \mathbf{W}(t), \\
d X_{j}^{(k)}(t)=X_{j}^{(k+1)}(t) d t, \\
X_{j}^{(0)}(t)=X_{j}(t)
\end{gathered}
$$

for $k=0,1, \ldots, n-2$. Since the instantaneous mean, $\mathbf{f}$, is an $N$-vector valued function, the index $j$ runs from one to $N$, that is, $j=1, \ldots, N$. The diffusion coefficent $\mathrm{G}$ is an $N \times M$ matrix valued function and $\mathbf{W}(t)$ is an $M$-dimensional standard Wiener process. From here onwards we denote $\left\{\mathbf{X}(t), \dot{\mathbf{X}}(t), \ldots, \mathbf{X}^{(n-1)}(t)\right\}$ by $\boldsymbol{X}^{(n-1)}(t)$. The context of this processes is that both the instantaneous drift and diffusion coefficients are Lipschitz continuous with respect to the right norm. A good example of the type of norm used for this is given by [7] in their seventh chapter.

The Lie point transformation methodology used by Wafo Soh and Mahomed [5] does all calculations to $\mathcal{O}(\theta)$. As a result, the recoverability of the finite transformations, which 
keep invariance, from the infinitesimal ones is not verified. The symmetry operator $H$ of point symmetry is

$$
H=\tau(x, t) \frac{\partial}{\partial t}+\xi_{j}(x, t) \frac{\partial}{\partial x_{j}}
$$

where there is summation $j=1, N$. However since we are dealing with an $n$ th-ordered SODE, prolongation formulation is necessary. In the Banach space, the transformation for the $(n-$ 1)th-ordered spatial derivative is

$$
\begin{aligned}
\overline{\mathbf{x}}^{(n-1)} & =e^{\theta H^{[n-1]}} x^{(n-1)}, \\
\overline{\mathbf{x}}^{(k)}= & e^{\theta H^{[n-1]}} x^{(k)} \\
= & e^{\theta H^{[k]}} x^{(k)}, \quad k<n-1,
\end{aligned}
$$

where

$$
\begin{gathered}
H^{[n-1]}=H^{(n-2)}+\xi_{j}^{[n-1]} \frac{\partial}{\partial x_{j}^{(n-1)}}, \quad n \geq 1, \\
H^{[0]}=H .
\end{gathered}
$$

Applying Itô's formula on an arbitrarily ordered prolongation of a spatial infinitesimal, $\xi_{j}^{[r]}\left(t, x^{(r-1)}(t)\right)$, gives

$$
d \xi_{j}^{[r]}\left(t, X^{(r-1)}(t)\right)=f_{\left(\xi^{[r]}\right) j}\left(t, X^{(r-1)}(t)\right) d t+G_{(\xi[r]) j}^{l}\left(t, X^{(r-1)}(t)\right) d W_{l}(t),
$$

where

$$
\begin{aligned}
f_{\left(\xi^{[r]}\right) j}\left(t, x^{(r-1)}(t)\right)= & \frac{\partial \xi_{j}^{[r]}}{\partial t}+f_{i} \frac{\partial \xi_{j}^{[r]}}{\partial x_{i}^{(n-1)}}+\frac{1}{2} \sum_{k=1}^{M} G_{i}^{k} G_{p}^{k} \frac{\partial^{2} \xi_{j}^{[r]}}{\partial x_{i}^{(n-1)} \partial x_{p}^{(n-1)}} \\
& +\sum_{\alpha=0}^{n-2} x_{p}^{(\alpha+1)} \frac{\partial \xi_{j}^{[r]}}{\partial x_{p}^{(\alpha)}}, \quad \text { where } n \geq 2 ; \\
G_{\left(\xi^{[r]}\right) j}^{l}\left(t, x^{(r-1)}(t)\right)= & \frac{\partial \xi_{j}^{[r]}}{\partial x_{i}^{(n-1)}} G_{i}^{l} r, \quad \text { for each } j \text { ranging from } 1 \text { to } N .
\end{aligned}
$$

If the summation operator runs from a nonnegative value, for example, 0 , to a negative one; that is, -1 , the outcome of the entire summation is set to zero. With this convention, we are able to recover the Itô formula for first-order SODEs. Due to the repeated index summation convention, the spatial indices $i$ and $p$ both run from 1 to $N$ in the summation; the Wiener 
indices $l$ and $k$ run from 1 to $M$. Similarly, the Itô's formula for the temporal variable, $\tau(x, t)$, gives

$$
d \tau=f_{(\tau)}(\mathbf{X}(t, \omega), t) d t+G_{(\tau)}^{l}(\mathbf{X}(t, \omega), t) d W_{l}(\mathbf{X}(t, \omega), t)
$$

where

$$
f_{(\tau)}(\mathbf{X}(t, \omega), t)=\frac{\partial \tau}{\partial t}+f_{i} \frac{\partial \tau}{\partial x_{i}^{(n-1)}}+\frac{1}{2} \sum_{k=1}^{M} G_{i}^{k} G_{p}^{k} \frac{\partial^{2} \tau}{\partial x_{i}^{(n-1)} \partial x_{p}^{(n-1)}}+\sum_{\alpha=0}^{n-2} x_{p}^{(\alpha+1)} \frac{\partial \tau}{\partial x_{p}^{(\alpha)}}
$$

which reduces to the total derivative, since the temporal infinitesimal is a point transformation

$$
f_{(\tau)}(\mathbf{X}(t, \omega), t)=D(\tau)
$$

where the total derivative is defined as

$$
D=\frac{\partial}{\partial t}+\sum_{\alpha=0}^{n-2} x_{p}^{(\alpha+1)} \frac{\partial}{\partial x_{p}^{(\alpha)}}
$$

The diffusion coefficient of the temporal infinitesimal is given by

$$
G_{(\tau)}^{l}(\mathbf{X}(t, \omega), t)=G_{i}^{l} \frac{\partial \tau}{\partial x_{i}^{(n-1)}}
$$

reduces to zero as well because of the fact that we are dealing with point transformations, that is,

$$
G_{(\tau)}^{l}(\mathbf{X}(t, \omega), t)=0
$$

The drift and diffusion coefficients of the $(n-1)$ th-order spatial derivative are, respectively, transformed as

$$
\begin{aligned}
& f_{j}\left(\bar{X}^{(n-1)}(\bar{t}), \bar{t}\right)=f_{j}\left(X^{(n-1)}(t), t\right)+\theta H^{[n-1]}\left(f_{j}\left(X^{(n-1)}(t), t\right)\right)+\mathcal{O}\left(\theta^{2}\right) \\
& G_{j}^{l}\left(\bar{x}^{(n-1)}(\bar{t}), \bar{t}\right)=G_{j}^{l}\left(x^{(n-1)}(t), t\right)+\theta H^{[n-1]}\left(G_{j}^{l}\left(x^{(n-1)}(t), t\right)\right)+\mathcal{O}\left(\theta^{2}\right) .
\end{aligned}
$$

The Itô formula of the finite time index transformation is

$$
d \bar{t}=\Gamma\left(e^{\theta H^{[n-1]}}(t)\right) d t+Y^{l}\left(e^{\theta H^{[n-1]}}(t)\right) d W_{l}
$$


which Wafo Soh and Mahomed [5] simply write as

$$
d \bar{t}=d t(1+\theta D(\tau))+\mathcal{O}\left(\theta^{2}\right)
$$

since the temporal infinitesimal is a point transformation. We also have that the transformed time index should keep invariance in the following probabilistic way:

$$
\mathbb{E}_{\overline{\mathbb{Q}}}[d \bar{t}(t, \omega)]=d \bar{t}(t, \omega)
$$

This requires

$$
Y^{l}\left(e^{\theta H^{[n-1]}}(t)\right)=0, \quad l=1, M
$$

which is automatically satisfied since $\tau$ is point transformation. Condition (2.19) also forces

$$
D\left(e^{\theta H^{[n-1]}}(t)\right)=\text { Constant }
$$

which is overlooked in [5]. Thus the finite transformation of the Wiener process is

$$
d \bar{W}_{l}(\bar{t}, \omega)=\sqrt{D\left(e^{\theta H^{[n-1]}}(t)\right)} d W_{l}(t, \omega)
$$

which Wafo Soh and Mahomed [5] simplify as

$$
d \bar{W}_{l}(\bar{t}, \omega)=d W_{l}(t, \omega)\left(1+\frac{\epsilon}{2} D(\tau)\right)+\mathcal{O}\left(\theta^{2}\right)
$$

where [5] used a generalized binomial expansion of the square-root of the derivative of the transformed time index with respect to time. The Itô SODE associated with Lie point $n$ thordered spatial transformation is

$$
d \bar{X}_{j}^{(n-1)}(\bar{t})=d X_{j}^{(n-1)}(t)+\theta\left(f_{\left(\xi^{[n-1]}\right) j} d t+G_{\left(\xi^{[n-1]}\right) j}^{l} d W_{l}(t)\right)+\mathcal{O}\left(\theta^{2}\right)
$$

Wafo Soh and Mahomed [5] make the assumption that only the system of $n$th order SODEs, (2.1), remains invariant under the symmetry operator (2.4), which implies that

$$
d \bar{X}_{j}^{(n-1)}(\bar{t})=f_{j}\left(\bar{t}, \bar{x}^{(n-1)}(\bar{t}, \omega)\right) d \bar{t}+G_{j}^{l}\left(\bar{t}_{,} \bar{x}^{(n-1)}(\bar{t}, \omega)\right) d \bar{W}_{l}(\bar{t})
$$

where we denote $\left\{\overline{\mathbf{X}}(\bar{t}), \overline{\mathbf{X}}(\bar{t}), \ldots, \overline{\mathbf{X}}^{(r-1)}(\bar{t})\right\}$ by $\bar{x}^{(r-1)}(\bar{t})$ for an arbitrary $r \in \mathbb{N}$. 

gives

Expanding the drift component $f_{j}\left(\bar{t}, \bar{x}^{(n-1)}(\bar{t}, \omega)\right) d \bar{t}$ of (2.25) using (2.15) and (2.18)

$$
\begin{array}{r}
\mathbf{f}\left(t, x^{(n-1)}(t)\right) d \bar{t}=\left\{\mathbf{f}\left(t, x^{(n-1)}(t)\right)+\theta\left(D(\tau)+H^{[n-1]}\right) \mathbf{f}\left(t, x^{(n-1)}(t)\right)\right. \\
+\sum_{k=2}^{\infty} \frac{\theta^{k}}{k !}\left(\left(D(\tau)+H^{[n-1]}\right)^{k} \mathbf{f}\left(t, x^{(n-1)}(t)\right)\right. \\
+\sum_{j=0}^{k-2}\left(\begin{array}{c}
k \\
k-j
\end{array}\right) H^{j}\left(\mathbf{f}\left(t, x^{(n-1)}(t)\right)\right) \\
\left.\left.\times\left(D\left(H^{k-j}(t)\right)-[D(\tau)]^{k-j}\right)\right)\right\} d t .
\end{array}
$$

In order for the finite transformations to keep invariance, we require a higher ordered $\theta$-terms to be solely dependent on the $\mathcal{O}(1)$ and $\mathcal{O}(\theta)$ terms, this forces the condition

$$
e^{\theta D(\tau)}=D\left(e^{\theta H^{[n-1]}}(t)\right)
$$

which is satisfied as a result of condition (2.21). Whence the finite transformation of the Wiener process becomes

$$
d \bar{W}_{l}(\bar{t}, \omega)=e^{\theta D(\tau) / 2} d W_{l}(t, \omega)
$$

The diffusion component of (2.25) can easily be expanded with the utility of (2.16) and (2.23)

$$
\begin{aligned}
G_{j}^{l}\left(t, X^{(n-1)}(t)\right) d \bar{W}_{l}=\{ & G_{j}^{l}\left(t, x^{(n-1)}(t)\right)+\theta\left(\frac{D(\tau)}{2}+H^{[n-1]}\right) G_{j}^{l}\left(t, X^{(n-1)}(t)\right) \\
& \left.+\sum_{k=2}^{\infty} \frac{\theta^{k}}{k !}\left(\frac{D(\tau)}{2}+H^{[n-1]}\right)^{k} G_{j}^{l}\left(t, x^{(n-1)}(t)\right)\right\} d W_{l} .
\end{aligned}
$$

This allows us to make a comparison with the Itô SODE associated with the $n$ th-ordered spatial transformation (2.24), which furnishes the determining equations used by Wafo Soh and Mahomed [5], that is,

$$
\begin{gathered}
f_{\left(\xi^{[n-1]}\right) j}=\left(D(\tau)+H^{[n-1]}\right) f_{j}\left(x^{(n-1)}(t), t\right), \\
G_{\left(\xi^{[n-1]}\right) j}^{l}=\left(\frac{D(\tau)}{2}+H^{[n-1]}\right) G_{j}^{l}\left(\bar{x}^{(n-1)}(\bar{t}), \bar{t}\right) .
\end{gathered}
$$


Constructing the prolonged variables was carried out in [5] by using preexisting recursive relations based on the Lie point theory for ODEs, that is,

$$
\xi_{j}^{[k]}=D \xi_{j}^{[k-1]}-x_{j}^{(k)} D_{\tau}, \quad \xi_{j}^{[0]}=\xi_{j}
$$

for $k \leq n$. The sketch of the methodology used for Lie point symmetries for $n$ th-ordered SODEs by Wafo Soh and Mahomed [5] ends here. However, it is possible to construct the recursive relations using form invariance arguments on the SODEs described in (2.2), that is,

$$
d \bar{X}_{j}^{(k)}(\bar{t})=\bar{X}^{(k+1)}(\bar{t}) d \bar{t}
$$

which after expanding yields the following $\theta$-ordered relations:

$$
\begin{gathered}
d \bar{X}_{j}^{(k)}(\bar{t})=X^{(k+1)} d t+\theta\left(\xi_{j}^{[k+1]}+x_{j}^{(k+1)} D(\tau)\right) d t+\mathcal{O}\left(\theta^{2}\right) \\
\Gamma\left(\xi^{[k]}\right)=\left(\Gamma(\tau)+H^{[k+1]}\right) X_{i}^{(k+1)}, \\
G_{i}^{j} \frac{\partial \xi^{[k]}}{\partial x_{i}^{(n-1)}}=0, \quad \text { for } k<n-1
\end{gathered}
$$

a new condition, which is not mentioned in [5], which is automatically satisfied since the terms $\xi^{[k]}$, where $k<n-1$, are not functions of $x^{(n-1)}$. In conjunction with this, we have the Itô SODE associated with the transformation of the $k$ th-ordered spatial transformation, that is,

$$
d \bar{X}_{j}^{(k)}(\bar{t})=d X_{j}^{(k)}(t)+\theta\left(f_{\left(\xi^{[k]}\right) j} d t+G_{\left(\xi^{[k]}\right) j}^{l} d W_{l}(t)\right)+\mathcal{O}\left(\epsilon^{2}\right)
$$

which reduces to

$$
d \bar{X}_{j}^{(k)}(\bar{t})=d X_{j}^{(k)}(t)+\theta D\left(\xi_{j}^{[k]}\right) d t+\mathcal{O}\left(\theta^{2}\right)
$$

as a result of the fact that the lower ordered prolongation infinitesimals $\xi_{j}^{[k]}$, are not a function of $\mathbf{x}^{(n-1)}$ for $k<(n-1)$. Thus the recursive relations, defined by Wafo Soh and Mahomed [5] from an ODE context, are easily derived using a form invariance philosophy, namely

$$
D\left(\xi_{j}^{[k]}\right)=\xi_{j}^{[k+1]}+x_{j}^{(k+1)} D(\tau)
$$

We now adapt the relations (2.30), (2.31), and (2.35) to an approximate SODE. 


\section{Symmetries of $n$th Order Multidimensional Approximate Stochastic Ordinary Differential Equations}

We now consider the following:

$$
\begin{gathered}
d \mathbf{X}^{(n-1)}(\bar{t})=\mathbf{f}\left(t, \mathbf{X}(t), \dot{\mathbf{X}}(t), \ldots, \mathbf{X}^{(n-1)}(t), R_{\mu}\right) d t+\cdots+\mathrm{G}\left(t, \mathbf{X}(t), \dot{\mathbf{X}}(t), \ldots, \mathbf{X}^{(n-1)}(t), R_{v}\right) d \mathbf{W}(t), \\
d X_{i}^{(k)}(t)=X_{i}^{(k+1)} d t, \\
X_{i}^{(0)}(t)=X_{i}(t)
\end{gathered}
$$

for $k=0,1, \ldots, n-2$. The function $\mathbf{f}$ is an approximate drift, which is an $N$ vector-valued function, $i=1, \ldots, N$. G is an $N \times M$ matrix-valued function approximating diffusion and $\mathbf{W}(t)$ is an $M$-dimensional Wiener process. Here $\mathbf{f}$ and $\mathbf{G}$ are defined as follows:

$$
\mathbf{f}\left(t, \mathbf{x}(t), \dot{\mathbf{x}}(t), \ldots, \mathbf{x}^{(n-1)}(t), R_{\mu}\right)=\epsilon^{r \mu} \mathbf{f}^{r}\left(t, \dot{\mathbf{x}}(t), \ldots, \mathbf{x}^{(n-1)}(t)\right)
$$

where the repeated index $r$ runs from 0 to $R_{\mu}$, where $R_{\mu}$ is the largest positive integer such that $\mu R_{\mu}<2 \rho$ and

$$
\mathbf{G}\left(t, \mathbf{x}(t), \dot{\mathbf{x}}(t), \ldots, \mathbf{x}^{(n-1)}(t), R_{v}\right)=\epsilon^{r v} \mathbf{G}^{r}\left(t, \mathbf{x}(t), \dot{\mathbf{x}}(t), \ldots, \mathbf{x}^{(n-1)}(t)\right)
$$

where the repeated index $r$ runs from 0 to $R_{v} ; R_{v}$ is the largest positive integer such that $v R_{v}<2 \rho$. The order of accuracy to which we choose to work is $\rho$.

The spatial and temporal variables of our infinitesimal generator

$$
H=\tau(t, \mathbf{x}, \rho) \frac{\partial}{\partial t}+\xi_{j}(t, \mathbf{x}, \rho) \frac{\partial}{\partial x_{j}}
$$

are defined as

$$
\begin{aligned}
& \tau(t, \mathbf{x}, \rho)=\epsilon^{r} \tau^{r}(t, \mathbf{x}), \\
& \xi(t, \mathbf{x}, \rho)=\epsilon^{r} \xi^{r}(t, \mathbf{x}) .
\end{aligned}
$$


The repeated index runs from 0 to $\rho$, since throughout this paper we will be working on $O\left(\epsilon^{\rho}\right)$. Using Itô's formula on the $(n-1)$ th-prolongation of the spatial, we get

$$
\begin{aligned}
d \xi_{j}^{[n-1]}= & \left(\frac{\partial \xi_{j}^{[n-1]}}{\partial t}+f_{i} \frac{\partial \xi_{j}^{[n-1]}}{\partial x_{i}^{(n-1)}}+\frac{1}{2} \sum_{s=1}^{M} G_{i}^{s} G_{k}^{s} \frac{\partial^{2} \xi_{j}^{[n-1]}}{\partial x_{i}^{(n-1)} \partial x_{k}^{(n-1)}}+\sum_{\alpha=0}^{n-2} x_{k}^{(\alpha+1)} \frac{\partial \xi_{j}^{[n-1]}}{\partial x_{k}^{(\alpha)}}\right) d t \\
& +\cdots+\frac{\partial \xi_{j}^{[n-1]}}{\partial x_{i}^{(n-1)}} G_{i}^{k} d W_{k}(t) \\
= & \left(\left(\frac{1}{2} \sum_{s=1}^{M} G_{i}^{0 s} G_{k}^{0 s} \frac{\partial^{2} \xi_{j}^{l[n-1]}}{\partial x_{i}^{(n-1)} \partial x_{k}^{(n-1)}}+f_{i} \frac{\partial \xi_{j}^{l[n-1]}}{\partial x_{i}}+\frac{\partial \xi_{j}^{[n-1]}}{\partial t}+\sum_{\alpha=0}^{n-2} x_{k}^{(\alpha+1)} \frac{\partial \xi_{j}^{[n-1]}}{\partial x_{k}^{(\alpha)}}\right) \epsilon^{l}\right. \\
& +\cdots+\frac{1}{2} \sum_{s=1}^{M} G_{i}^{p s} G_{k}^{p s} \frac{\partial^{2} \xi_{j}^{l[n-1]}}{\partial x_{i}^{(n-1)} \partial x_{k}^{(n-1)}} \epsilon^{l+2 p v}+\sum_{s=1}^{M} G_{i}^{r s} G_{k}^{p s} \frac{\partial^{2} \xi_{j}^{l[n-1]}}{\partial x_{i}^{(n-1)} \partial x_{k}^{(n-1)}} \epsilon^{l+v(r+p)} \\
& \left.+\cdots+f_{i} \frac{\partial \xi_{j}^{l[n-1]}}{\partial x_{i}^{(n-1)}} \epsilon^{l+\mu q}\right) d t+\cdots+G_{i}^{p s} \frac{\partial \xi_{j}^{l[n-1]}}{\partial x_{s}^{(n-1)}} \epsilon^{l+v p} d W^{(i)}
\end{aligned}
$$

and on the temporal infinitesimal

$$
\begin{aligned}
d \tau & =D(\tau) d t \\
& =\epsilon^{l}\left(\frac{\partial \tau^{l}}{\partial t}+\sum_{\alpha=0}^{n-2} x_{k}^{(\alpha+1)} \frac{\partial \tau^{l}}{\partial x_{k}^{(n-1)}}\right) d t
\end{aligned}
$$

with

$$
\stackrel{p}{G}_{i}^{s} \frac{\partial \tau^{l}}{\partial x_{s}^{(n-1)}} \epsilon^{l+v p}=0
$$

which is automatically satisfied since $\tau$ is point symmetry. The repeated indices $r, p, q$, and $l$ run from 0 to $R_{v}-1, R_{v}, R_{\mu}$, and $\rho$, respectively in our repeated index summation convention; $r<p$. Thus, by substitution, we get

$$
\begin{array}{r}
d \bar{X}^{(n-1)}=d X^{(n-1)}+\cdots+\theta\left(\frac{\partial \xi^{[n-1]}}{\partial t}+f_{i} \frac{\partial \xi^{[n-1]}}{\partial x_{i}^{(n-1)}}+\frac{1}{2} \sum_{k=1}^{M} G_{i}^{k} G_{j}^{k} \frac{\partial^{2} \xi^{[n-1]}}{\partial x_{i}^{(n-1)} \partial x_{j}^{(n-1)}}\right. \\
\left.+\sum_{\alpha=0}^{n-2} x_{j}^{(\alpha+1)} \frac{\partial \xi^{[n-1]}}{\partial x_{j}^{(\alpha)}}\right) d t+\theta G_{j}^{i} \frac{\partial \xi^{[n-1]}}{\partial x_{i}^{(n-1)}} d W_{t}^{(j)}+\mathcal{O}\left(\theta^{2}\right)
\end{array}
$$




$$
\begin{gathered}
d \bar{t}=d t+\theta D(\tau) d t+\mathcal{O}\left(\theta^{2}\right) \\
d \bar{W}_{\bar{t}}=d W_{t}^{(l)}\left(1+\frac{\theta}{2} D(\tau)\right)+\mathcal{O}\left(\theta^{2}\right) .
\end{gathered}
$$

The transformation of $\mathbf{f}$ and $\mathbf{G}$ under our prolongated infinitesimal generator $H^{[\beta]}$ is

$$
\begin{aligned}
f_{i}\left(\bar{t}, \hat{x}^{(n-1)}\right) & =f_{i}\left(t, x^{(n-1)}\right)+\theta H^{[\beta]} f_{i}\left(t, x^{(n-1)}\right)+\mathcal{O}\left(\theta^{2}\right) \\
& =f_{i}+\theta\left(\tau(t, \dot{\mathbf{X}}(t)) \frac{\partial f_{i}}{\partial t}+\xi_{j}^{[\beta]}\left(t, x^{(n-1)}\right) \frac{\partial f_{i}}{\partial x_{j}^{(\beta)}}\right)+\mathcal{O}\left(\theta^{2}\right) \\
& =\epsilon^{q} f_{i}^{q}+\theta \xi_{j}^{l[\beta]} \frac{\partial f_{i}^{q}}{\partial x_{j}^{(\beta)}} \epsilon^{l+\mu q}+\theta \tau^{l} \frac{\partial f_{i}^{q}}{\partial t} \epsilon^{l+\mu q}+\mathcal{O}\left(\theta^{2}\right) \\
& =\epsilon^{q} f_{i}^{q}+\theta \epsilon^{l+\mu q}\left(\xi_{j}^{l[\beta]} \frac{\partial f_{i}^{q}}{\partial x_{j}^{(\beta)}}+\tau^{l} \frac{\partial f_{i}^{q}}{\partial t}\right)+\mathcal{O}\left(\theta^{2}\right) \\
G_{k}^{i}\left(\bar{t}, \widehat{x}^{(n-1)}\right) & =G_{k}^{i}\left(t, x^{(n-1)}\right)+\theta H^{[\beta]} G_{k}^{i}\left(t, x^{(n-1)}\right)+\mathcal{O}\left(\theta^{2}\right) \\
& =G_{k}^{i}+\theta\left(\tau(t, \dot{\mathbf{X}}(t)) \frac{\partial G_{k}^{i}}{\partial t}+\xi_{j}^{[\beta]}\left(t, x^{(n-1)}\right) \frac{\partial G_{k}^{i}}{\partial x_{j}^{(\beta)}}\right)+\mathcal{O}\left(\theta^{2}\right) \\
& =\epsilon^{p} G_{k}^{p i}+\theta \epsilon^{v p+l}\left(\xi_{j}^{l[\beta]} \frac{\partial G_{k}^{p i}}{\partial x_{j}^{(\beta)}}+\tau^{l} \frac{\partial G_{k}^{p i}}{\partial t}\right)+\mathcal{O}\left(\theta^{2}\right),
\end{aligned}
$$

where $\left\{X, \dot{X}, \ldots, X^{(n-1)}\right\}$ is represented by $X^{(n-1)}$ and the transformed set $\left\{\bar{X}, \bar{X}, \ldots, \overline{X^{(n-1)}}\right\}$ is represented by $\widehat{X}^{(n-1)}$. The repeated indices $q, p, l$, and $n$ run from 0 to $R_{\mu}, R_{v}, \rho$, and $n-1$, respectively.

\subsection{Operators}

Thus the determining equations (2.30), (2.31), and (2.35) become

$$
\epsilon^{\mu q+l}\left(f_{m}^{q}\left(\Gamma^{0}\left(\tau^{l}\right)+\epsilon^{v(r+p)} \mho_{r p}\left(\tau^{l}\right)+\epsilon^{2 v p} \Upsilon_{p}\left(\tau^{l}\right)+\epsilon^{\mu j} \Psi_{j}\left(\tau^{l}\right)\right)+H_{\beta}^{l}\left(f_{m}^{q}\right)\right)
$$


Journal of Applied Mathematics

$$
\begin{gathered}
+\cdots-\epsilon^{l}\left(\Gamma^{0}\left(\xi_{m}^{l[n-1]}\right)+\epsilon^{v(r+p)} \mho_{r p}\left(\xi_{m}^{l[n-1]}\right)\right. \\
\left.+\epsilon^{2 v p} \Upsilon_{p}\left(\xi_{m}^{l[n-1]}\right)+\epsilon^{\mu q} \Psi_{q}\left(\xi_{m}^{l[n-1]}\right)\right)=0, \\
\epsilon^{l+v p}\left(H_{\beta}^{l}\left(G_{k}^{p m}\right)+\frac{1}{2} G_{k}^{p m}\right. \\
\left.\times\left(\Gamma^{0}\left(\tau^{l}\right)+\epsilon^{v(r+p)} \mho_{r p}\left(\tau^{l}\right)+\epsilon^{2 v p} \Upsilon_{p}\left(\tau^{l}\right)+\epsilon^{\mu q} \Psi_{q}\left(\tau^{l}\right)\right)-Y_{n-1}^{p k}\left(\xi_{m}^{l[n-1]}\right)\right)=0, \\
\epsilon^{l+v p} \Upsilon_{n-1}^{p i}\left(\tau^{l}\right)=0, \\
\epsilon^{l+v p} \Upsilon_{n-1}^{p i}\left(\xi_{i}^{l[k]}\right)=0, \quad k<n-1, \\
\epsilon^{l}\left(\Gamma^{0}\left(\xi_{m}^{l[k]}\right)+\epsilon^{v(r+p)} \mho_{r p}\left(\xi_{m}^{[l k]}\right)+\epsilon^{2 v p} \Upsilon_{p}\left(\xi_{m}^{l[k]}\right)+\epsilon^{\mu q} \Psi_{q}\left(\xi_{m}^{l[k]}\right)\right) \\
=\epsilon^{l}\left(x_{i}^{(k+1)}\left(\Gamma^{0}\left(\tau^{l}\right)+\epsilon^{v(r+p)} \mho_{r p}\left(\tau^{l}\right)+\epsilon^{2 v p} \Upsilon_{p}\left(\tau^{l}\right)+\epsilon^{\mu j} \Psi_{j}\left(\tau^{l}\right)\right)+\xi_{i}^{l[k+1]}\right),
\end{gathered}
$$

respectively, where

$$
\begin{aligned}
& \Gamma^{0}=\frac{1}{2} \sum_{s=1}^{M} G_{i}^{0 s} G_{k}^{0 s} \frac{\partial^{2}}{\partial x_{i}^{(n-1)} \partial x_{k}^{(n-1)}}+f_{i}^{0} \frac{\partial}{\partial x_{i}^{(n-1)}}+\frac{\partial}{\partial t}+\cdots+\sum_{\alpha=0}^{n-1-1} x_{k}^{(\alpha+1)} \frac{\partial}{\partial x_{k}^{(n-1)}}, \\
& \mho_{r p}=\sum_{s=1}^{M} G_{i}^{r s} G_{k}^{p s} \frac{\partial^{2}}{\partial x_{i}^{(n-1)} \partial x_{k}^{(n-1)}}, \quad\left(0 \leq r<p \leq R_{v}\right), \\
& \Upsilon_{p}=\frac{1}{2} \sum_{s=1}^{M} G_{i}^{p s} G_{k}^{p s} \frac{\partial^{2}}{\partial x_{i}^{(n-1)} \partial x_{k}^{(n-1)}}, \quad\left(0<p \leq R_{v}\right), \\
& \Psi_{q}=f_{i}^{q} \frac{\partial}{\partial x_{i}^{(n-1)}}, \quad\left(0<q, j \leq R_{\mu}\right), \\
& Y_{n-1}^{p i}=G_{i}^{p k} \frac{\partial}{\partial x_{k}^{(n-1)}}, \quad\left(0 \leq p \leq R_{v}\right), \\
& H_{\beta}^{l}=\tau^{l} \frac{\partial}{\partial t}+\xi_{j}^{l[\beta]} \frac{\partial}{\partial x_{j}^{(\beta)}}, \quad(0 \leq \beta \leq n-1) .
\end{aligned}
$$

Note that we cannot cancel out the terms $\epsilon^{l}$ and $\epsilon^{l+v p}$ in (3.11) and (3.12), respectively, in order to simplify them. These terms are a part of the summation convention implied by the repeated indices. These terms contribute to the order of error as a result of this implication. 
We now apply our generalized methodology for finding approximate symmetries to the Itô system considered in [3]. Our application should be consistent with the determining equations found in Ibragimov et al. [3].

\subsubsection{Example 1}

For their approximate stochastic ordinary differential equations, $n-1=0, \mu=1, v=1 / 2$, $R_{\mu}=1, R_{v}=1$, and $\rho=1$. Thus the diffusion coefficient $\mathrm{G}$, which was taken to be constant, and the drift $\mathbf{f}$ appeared as follows in the Itô system:

$$
d \mathbf{x}=\left(\mathbf{f}^{0}+\epsilon \mathbf{f}^{1}\right) d t+\sqrt{\epsilon} \mathbf{G} d \mathbf{W}_{t}
$$

where the drift is a $N \times 1$ vector and the constant diffusion coefficient is a matrix with dimension $N \times M$. The determining equations are

$$
\begin{gathered}
-\frac{1}{2} \sum_{s=1}^{M} G_{k}^{1 s} G_{i}^{1 s} \epsilon\left(\frac{\partial^{2} \xi_{j}^{0}}{\partial x_{i} \partial x_{k}}+\epsilon \frac{\partial^{2} \xi_{j}^{1}}{\partial x_{i} \partial x_{k}}\right)+\left(\xi_{i}^{0}+\epsilon \xi_{i}^{1}\right)\left(\frac{\partial f_{j}^{0}}{\partial x_{i}}+\epsilon \frac{\partial f_{j}^{1}}{\partial x_{i}}\right)-f_{i}^{0}\left(\frac{\partial \xi_{j}^{0}}{\partial x_{i}}+\epsilon \frac{\partial \xi_{j}^{1}}{\partial x_{i}}\right) \\
-\epsilon f_{i}^{1}\left(\frac{\partial \xi_{j}^{0}}{\partial x_{i}}+\epsilon \frac{\partial \xi_{j}^{1}}{\partial x_{i}}\right)+\left(\tau^{0}+\epsilon \tau^{1}\right)\left(\frac{\partial f_{j}^{0}}{\partial t}+\epsilon \frac{\partial f_{j}^{1}}{\partial t}\right)-\frac{\partial \xi_{j}^{0}}{\partial t}-\epsilon \frac{\partial \xi_{j}^{1}}{\partial t}+\left(f_{j}^{0}+\epsilon f_{j}^{1}\right) \\
\times\left(\frac{1}{2} \epsilon \sum_{s=1}^{M} G_{k}^{1 s} G_{i}^{1 s}\left(\frac{\partial^{2} \tau^{0}}{\partial x_{i} \partial x_{k}}+\epsilon \frac{\partial^{2} \tau^{1}}{\partial x_{i} \partial x_{k}}\right)+f_{i}^{0}\left(\frac{\partial \tau^{0}}{\partial x_{i}}+\epsilon \frac{\partial \tau^{1}}{\partial x_{i}}\right)\right. \\
\left.+\epsilon f_{i}^{1}\left(\frac{\partial \tau^{0}}{\partial x_{i}}+\epsilon \frac{\partial \tau^{1}}{\partial x_{i}}\right)+\frac{\partial \tau^{0}}{\partial t}+\epsilon \frac{\partial \tau^{1}}{\partial t}\right)=0, \\
\times\left(\frac{1}{2} \epsilon\left(\frac{\partial^{2} \tau^{0}}{\partial x_{i} \partial x_{l}}+\epsilon \frac{\partial^{2} \tau^{1}}{\partial x_{i} \partial x_{l}}\right) \sum_{s=1}^{M} G_{i}^{1 s} G_{l}^{1 s}+f_{i}^{0}\left(\frac{\partial \tau_{k}^{1 j}}{\partial x_{i}}-\sqrt{\epsilon} G_{k}^{1 i}\left(\frac{\partial \xi_{j}^{0}}{\partial x_{i}}+\epsilon \frac{\partial \xi_{j}^{1}}{\partial x_{i}}\right)\right)+\sqrt{\epsilon}\left(\tau^{0}+\epsilon \tau^{1}\right) \frac{\partial G_{k}^{1 j}}{\partial t}+\frac{1}{2} \sqrt{\epsilon} G_{k}^{1 j}\right. \\
\left.+\epsilon f_{i}^{1}\left(\frac{\partial \tau^{0}}{\partial x_{i}}+\epsilon \frac{\partial \tau^{1}}{\partial x_{i}}\right)+\frac{\partial \tau^{0}}{\partial t}+\epsilon \frac{\partial \tau^{1}}{\partial t}\right)=0 .
\end{gathered}
$$

Now since we are working to order $\rho$, we get the following groups of determining equations which are exactly what Ibragimov et al. [3] get

$$
-\frac{\partial \xi_{j}^{0}}{\partial t}-f_{i}^{0} \frac{\partial \xi_{j}^{0}}{\partial x_{i}}+\xi_{i}^{0} \frac{\partial f_{j}^{0}}{\partial x_{i}}+\tau^{0} \frac{\partial f_{j}^{0}}{\partial t}+f_{j}^{0} f_{i}^{0} \frac{\partial \tau^{0}}{\partial x_{i}}+f_{j}^{0} \frac{\partial \tau^{0}}{\partial t}=0
$$


which we get by comparing coefficients with no $\epsilon^{\prime}$ s

$$
\begin{gathered}
f_{j}^{0} f_{i}^{0} \frac{\partial \tau^{1}}{\partial x_{i}}-\frac{\partial \xi_{j}^{1}}{\partial x_{i}} f_{i}^{0}+f_{j}^{0} f_{i}^{1} \frac{\partial \tau^{0}}{\partial x_{i}}+f_{j}^{1} f_{i}^{0} \frac{\partial \tau^{0}}{\partial x_{i}}+\frac{1}{2} \frac{\partial^{2} \tau^{0}}{\partial x_{i} \partial x_{l}} \sum_{s=1}^{M} G_{i}^{1 s} G_{l}^{1 s} f_{j}^{0}+\frac{\partial \tau^{1}}{\partial t} f_{j}^{0}+\xi_{i}^{1} \frac{\partial f_{j}^{0}}{\partial x_{i}}+\xi_{i}^{0} \frac{\partial f_{j}^{1}}{\partial x_{i}} \\
-f_{i}^{1} \frac{\partial \xi_{j}^{0}}{\partial x_{i}}-\frac{1}{2} \frac{\partial^{2} \xi_{j}^{0}}{\partial x_{i} \partial x_{l}} \sum_{s=1}^{M} G_{i}^{1 s} G_{l}^{1 s}+\frac{\partial f_{j}^{0}}{\partial t} \tau^{1}+\frac{\partial f_{j}^{1}}{\partial t} \tau^{0}-\frac{\partial \xi_{j}^{1}}{\partial t}+\frac{\partial \tau^{0}}{\partial t} f_{j}^{1}=0
\end{gathered}
$$

which all share the same coefficient $\epsilon$. In a similar fashion, we get the following for $\sqrt{\epsilon}$ and $\epsilon$, respectively

$$
\begin{gathered}
\frac{1}{2} G_{k}^{1 j}\left(\frac{\partial \tau^{0}}{\partial t}+f_{i}^{0} \frac{\partial \tau^{0}}{\partial x_{i}}\right)-G_{k}^{1 i} \frac{\partial \xi_{j}^{0}}{\partial x_{i}}=0 \\
-G_{k}^{1 i} \frac{\partial \xi_{j}^{1}}{\partial x_{i}}+\frac{1}{2} G_{k}^{1 j}\left(\frac{\partial \tau^{1}}{\partial t}+f_{i}^{0} \frac{\partial \tau^{1}}{\partial x_{i}}\right)+\frac{1}{2} G_{k}^{1 j} f_{i}^{1} \frac{\partial \tau^{0}}{\partial x_{i}}+\frac{G_{k}^{1 j}}{4} \sum_{s=1}^{M} G_{i}^{1 s} G_{l}^{1 s} \frac{\partial^{2} \tau^{0}}{\partial x_{i} \partial x_{l}}=0
\end{gathered}
$$

Notice that we used (2.21) and the fact that $G$ was constant to simplify the above.

Remark 3.1. Our application is consistent with the findings of [3] for this example.

\subsubsection{Example 2}

We consider

$$
d \dot{X}=-\omega^{2} X d t+\sigma d W+\sqrt{\epsilon} X d W
$$

By applying the condition (2.35), we have that

$$
\xi=\xi(t, x)
$$

and thus the prolongation formula (2.34) becomes

$$
\xi^{[1]}=D(\xi)-\dot{x} D(\tau)
$$

where $D$ is the total time derivative operator. Our determining equations at $\epsilon^{0}$ are

$$
\begin{gathered}
-\omega^{2} x \Gamma^{0}\left(\tau^{0}\right)+H^{0}\left(-\omega^{2} x\right)=\Gamma^{0}\left(\xi^{0[1]}\right), \\
H^{0}\left(G^{0}\right)+\frac{1}{2} G^{0} \Gamma^{0}\left(\tau^{0}\right)=Y^{0}\left(\xi^{0[1]}\right) .
\end{gathered}
$$


The determining equations at $\epsilon$ are

$$
\begin{gathered}
-\omega^{2} x \Gamma^{0}\left(\tau^{1}\right)+H^{1}\left(-\omega^{2} x\right)-\omega^{2} x \Upsilon_{1}\left(\tau^{0}\right)=\Gamma^{0}\left(\xi^{1[1]}\right)+\Upsilon_{1}\left(\xi^{0}\right), \\
H^{1}\left(G^{0}\right)+\frac{1}{2} G^{0} \Gamma^{0}\left(\tau^{1}\right)=\Upsilon^{0}\left(\xi^{[1]}\right) .
\end{gathered}
$$

At $\epsilon^{1 / 2}$ the determining equations are

$$
H^{0}\left(G^{1}\right)+\frac{1}{2} G^{1} \Gamma^{0}\left(\tau^{0}\right)+\frac{1}{2} G^{0} \mho_{10}\left(\tau^{0}\right)=Y^{1}\left(\xi^{0[1]}\right)
$$

and the final determining equation at $\epsilon^{3 / 2}$ is

$$
\frac{1}{2} G^{0} \mho_{12}\left(\tau^{0}\right)+\frac{1}{2} G^{1} \Gamma^{0}\left(\tau^{1}\right)=Y^{1}\left(\xi^{1[1]}\right)
$$

Equation (3.31) for the infinitesimals at the zeroth echelon, that is, $\tau^{0}$ and $\xi^{0}$, have been solved earlier in the oscillating-spring mass example

$$
\begin{gathered}
\tau^{0}=C_{0} \\
\xi^{0}=C_{1} \cos (\omega t)+C_{2} \sin (\omega t) .
\end{gathered}
$$

Whence, (3.34) and (3.35) force

$$
\begin{gathered}
\xi^{0}=0 \\
\tau^{1}=C_{3} .
\end{gathered}
$$

From (3.32), we get

$$
-\omega^{2} \xi^{1}=D^{2}\left(\xi^{1}\right)
$$

which solves as

$$
\xi^{1}=C_{4} \cos (\omega t)+C_{5} \sin (\omega t) .
$$

Therefore we have

$$
\begin{gathered}
\xi=\epsilon\left(C_{4} \cos (\omega t)+C_{5} \sin (\omega t)\right), \\
\tau=C_{0}+\epsilon C_{3} .
\end{gathered}
$$




\section{Concluding Comments}

Lie group analysis for $n$ th-ordered Itô SODEs was first pursued in Wafo Soh and Mahomed [5]. Though it had only been done for point symmetries, it has led to many interesting findings in this paper. We have shown that it is possible to derive the prolongation formulas by using the philosophy of form invariance.

With the use of the philosophy that the properties of the Wiener processes should remain invariant under the Lie group transformations, we derive conditions on the temporal and lower level derivative spatial infinitesimals that are a generalization of the condition derived by Ünal [4] for one-dimensional SODEs.

In this more general approximate approach to higher order SODE, we derive the same conditioning as Ünal [4] did without recourse to the Itô's multiplication table for the transformed variables. Our results are consistent with that of [3] in the first-order case. However, we have a generalization to $n$ th-order SODEs. We also applied our method to an example taken from [3] as well as another.

\section{References}

[1] E. Fredericks and F. M. Mahomed, "A formal approach for handling Lie point symmetries of scalar first-order Itô stochastic ordinary differential equations," Journal of Nonlinear Mathematical Physics, vol. 15, supplement 1, pp. 44-59, 2008.

[2] E. Fredericks and F. M. Mahomed, "An alternative "W-symmetries" approach to Lie point symmetries of scalar first-order itô stochastic ordinary differentialequations," In press.

[3] N. H. Ibragimov, G. Ünal, and C. Jogréus, "Group analysis of stochastic differential systems: approximate symmetries and conservation laws," ALGA, vol. 1, pp. 95-126, 2004.

[4] G. Ünal, "Symmetries of Itô and Stratonovich dynamical systems and their conserved quantities," Nonlinear Dynamics, vol. 32, no. 4, pp. 417-426, 2003.

[5] C. Wafo Soh and F. M. Mahomed, "Integration of stochastic ordinary differential equations from a symmetry standpoint," Journal of Physics A, vol. 34, no. 1, pp. 177-192, 2001.

[6] E. Fredericks and F. M. Mahomed, "Symmetries of first-order stochastic ordinary differential equations revisited," Mathematical Methods in the Applied Sciences, vol. 30, no. 16, pp. 2013-2025, 2007.

[7] Z. Brzeźniak and T. Zastawniak, Basic Stochastic Processes, Springer, 2002. 


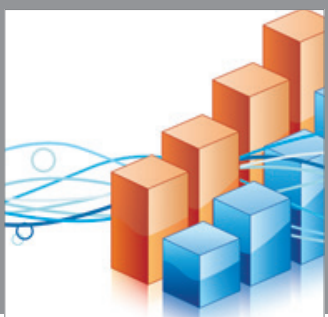

Advances in

Operations Research

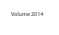

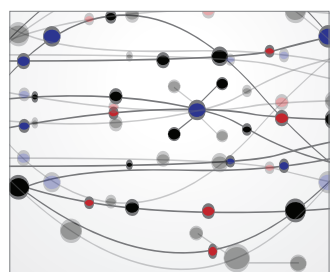

\section{The Scientific} World Journal
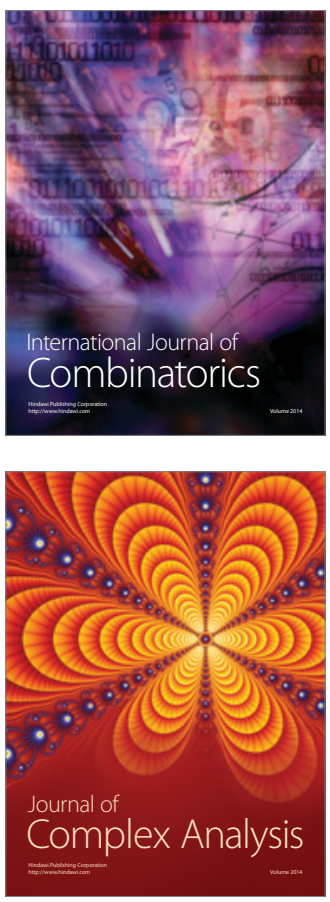

International Journal of

Mathematics and

Mathematical

Sciences
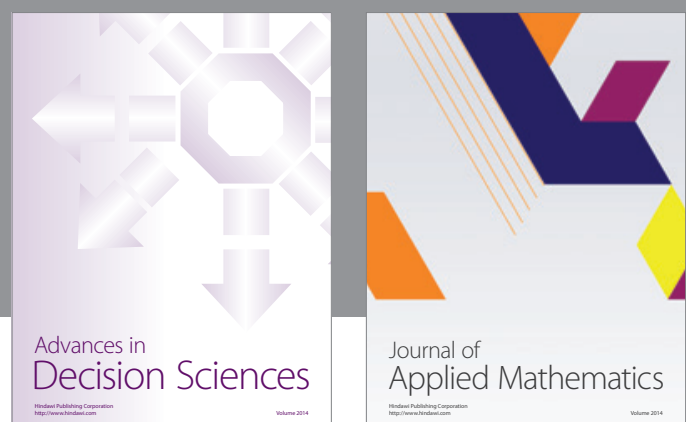

Journal of

Applied Mathematics
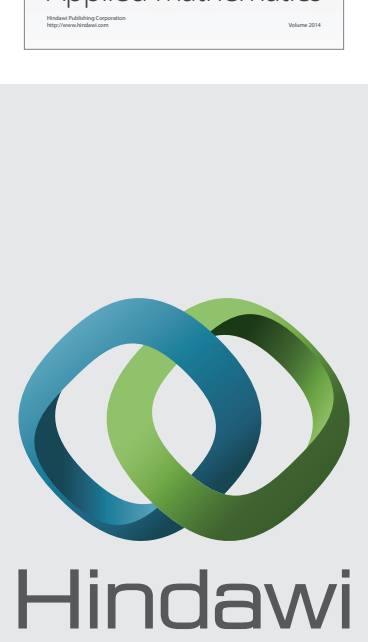

Submit your manuscripts at http://www.hindawi.com
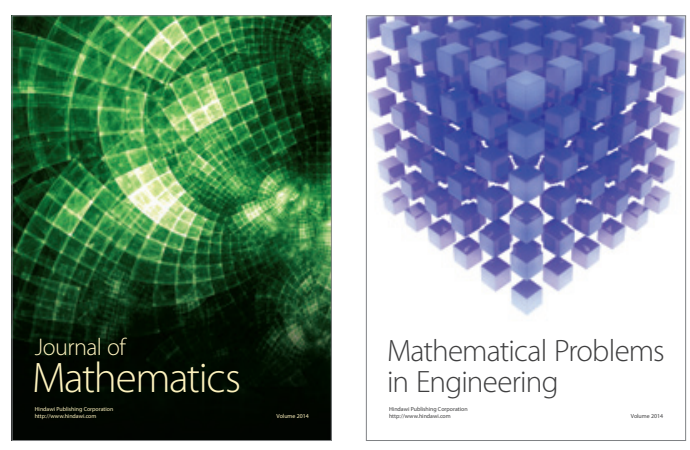

Mathematical Problems in Engineering
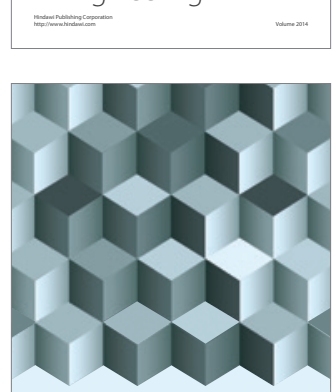

Journal of

Function Spaces
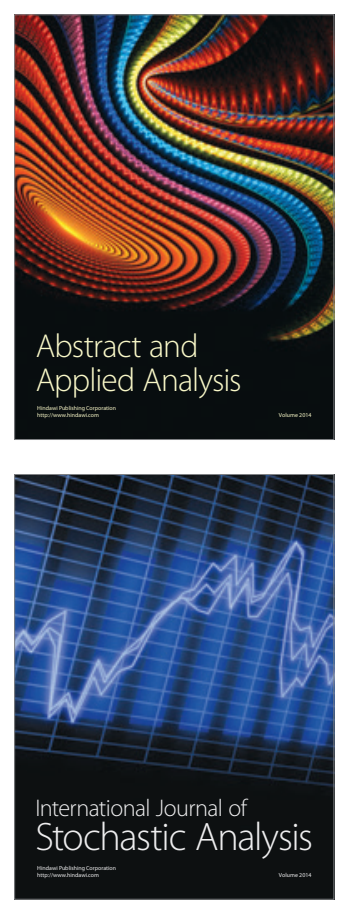

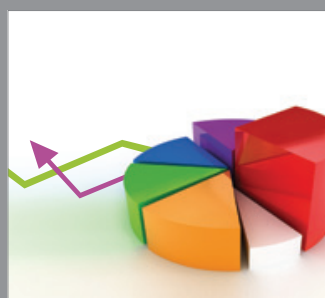

ournal of

Probability and Statistics

Promensencen
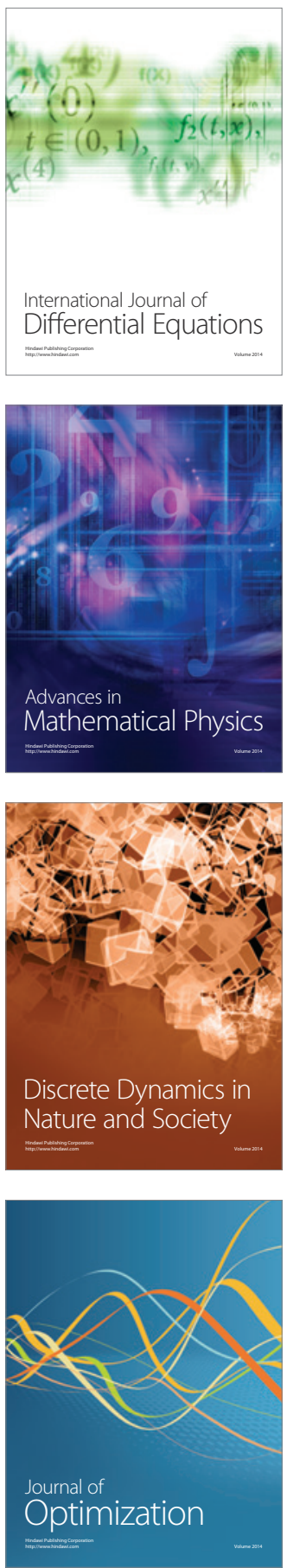